\title{
Vortex line density of superfluid suction vortex
}

Ken Obara, Itsuki Matsumura, Naoya Tajima, Katsuyoshi Ohyama, Hideo Yano, and

Osamu Ishikawa

\begin{tabular}{|c|l|}
\hline Citation & PHYSICAL REVIEW FLUIDS. 6(6); 064802 \\
\hline Issue Date & 2021-06-04 \\
\hline Type & Journal article \\
\hline Textversion & Author \\
\hline Rights & $\begin{array}{l}\text { C2021 American Physical Society. This article may be } \\
\text { downloaded for personal use only. Any other use requires prior } \\
\text { permission of the author and American Physical Society. } \\
\text { The following article appeared in } \\
\text { https://doi.org/10.1103/PhysRevFluids.6.064802. }\end{array}$ \\
\hline DOI & \begin{tabular}{l} 
10.1103/PhysRevFluids.6.064802 \\
\hline
\end{tabular} \\
\hline
\end{tabular}

\section{Self-Archiving by Author(s) \\ Placed on: Osaka City University Repository}

Obara, K., Matsumura, I., Tajima, N., Ohyama, K., Yano, H., \& Ishikawa, O.

(2021). Vortex line density of superfluid suction vortex. Physical Review Fluids.

Vol. 6, Issue 6. American Physical Society.

https://doi.org/10.1103/physrevfluids.6.064802 


\title{
Vortex line density of superfluid suction vortex
}

\author{
Ken Obara $\odot,{ }^{*}, \dagger$ Itsuki Matsumura, Naoya Tajima, Katsuyoshi Ohyama, \\ Hideo Yano ${ }^{\circ}$, and Osamu Ishikawa \\ Graduate School of Science, Osaka City University, 3-3-138 Sugimoto-cho, Sumiyoshi-ku, Osaka, Japan
}

(Received 23 November 2020; accepted 21 May 2021; published 4 June 2021)

\begin{abstract}
Although a suction vortex is the one of the most common vortices appearing in our everyday life, its internal structure has not been fully understood yet. One of the few things that we understand is that the radius of the core results from the competition between the diffusion of the vorticity due to the kinematic viscosity and the transportation of the vorticity due to the inward flow. To understand the structure of the suction vortex, we investigated a suction vortex produced in liquid helium. In the normal fluid phase, liquid helium behaves as a viscous fluid. In contrast, in the superfluid phase, a quantum vortex line carries all the microscopic circulation, and the suction vortex can be understood as a complex of quantized vortex lines. We generated a superfluid suction vortex with a cryogenic turbine and measured its circulation with a pulsed first sound circulation meter, and also measured the vorticity by the second sound attenuation technique. The experimental results indicate that the quantized vortex lines accumulated in the narrow region around the axis of symmetry due to an inward flow, the core structure of which cannot be described by a simple bundle of fully polarized quantized vortex lines.
\end{abstract}

DOI: 10.1103/PhysRevFluids.6.064802

\section{INTRODUCTION}

A vortex is defined as a region within a fluid in which the flow revolves around an axis line. Vortices can be formed in a wide range of scales, such as intergalaxy motion, atmospheric motion in planets, local tidal motion, draining flow in bathtub, stirring beverages, and quantized vortices in Bose-Einstein condensates and He II. In a classical viscous fluid, vortices can be classified into two types: vortices with vorticity and vortices without vorticity. Here vorticity is defined as $\boldsymbol{\omega}=\operatorname{rot} \boldsymbol{v}$, and $\boldsymbol{v}$ is the velocity field. The simplest way to generate a vortex with finite vorticity is to spin a liquid-filled cylindrical container at constant angular speed $\Omega$ about its vertical axis. In this case, because of the viscous effect, the liquid will rotate as a rigid body, the azimuthal velocity at radial position $r$ will become $v_{\theta}^{\mathrm{rb}}(r)=r \Omega$, and the vorticity will become $|\omega|=2 \Omega$.

The shape of the surface of the vortex will be deformed as $\Delta H(r)=r^{2} \Omega^{2} / 2 g$, where $g$ is the acceleration due to gravity. Vortices without vorticity, in contrast, are called free vortices, and their azimuthal velocity is inversely proportional to the radial position, that is, $v_{\theta}^{\mathrm{f}}(r)=\Gamma / 2 \pi r$, where $\Gamma$ is the circulation. However, the ideal free vortex has not yet been discovered in a viscous fluid because the speed of the liquid rapidly increases as it approaches the axis of rotation, which is energetically unfavorable. Therefore, a free vortex always has a finite velocity region near its center of rotation, and this is called the core of the vortex.

*k-obara@osaka-cu.ac.jp

${ }^{\dagger}$ Also at Nambu Institute for Experimental and Theoretical Physics, Osaka City University. 
The Burgers vortex [1] is a solvable vortex model that includes a vertical and inward flow, which is described by

$$
\boldsymbol{v} \equiv\left[v_{r}^{B}(r), v_{\theta}^{B}(r), v_{z}^{B}(z)\right], \quad v_{r}^{B}(r)=-\beta r, \quad v_{z}^{B}(z)=2 \beta z,
$$

where $\beta(>0)$ is the flow parameter that ensures the equation of continuity. The stationary solution of the Navier-Stokes equation for this stagnation flow gives the azimuthal velocity $v_{\theta}^{B}(r)$ and the vertical component of the vorticity $\omega_{z}^{B}(r)$ as

$$
v_{\theta}^{B}(r)=\frac{\Gamma}{2 \pi r}\left[1-\exp \left(-\frac{\beta r^{2}}{2 v}\right)\right], \quad \omega_{z}^{B}(r)=\frac{\Gamma \beta}{2 \pi v} \exp \left(-\frac{\beta r^{2}}{2 v}\right),
$$

where $v$ is the kinematic viscosity of the fluid. The flow profile shows a maximum at $r_{\mathrm{c}}=\sqrt{2 \nu \vartheta / \beta}$, where $\vartheta=1.2564312 \ldots$ is a solution of $2 \vartheta=e^{\vartheta}-1$. The core radius of this vortex is understood to result from the balance between the vorticity diffusion due to the viscosity and the vorticity transportation due to the inward flow.

A suction vortex is a type of free vortex that has a vertical suction flow that is going down toward a drain hole. Although it is the one of the most common vortices that appear in our everyday life $[2,3]$, neither the internal structure nor the flow field have yet been fully understood. The main reasons for this are the experimental difficulty due to the very rapid change of the flow in the core, and the lack of a direct technique to measure the vorticity in such a high-speed region. Roughly speaking, the structure of a stationary and axisymmetric suction vortex can be divided into two regions. The first region is the so-called potential flow region that does not have a vorticity and where the potential flow is revolving around the core. In the potential flow region, the azimuthal velocity at the point far enough away from the core can be described as

$$
v_{\theta}(r)=\frac{\Gamma}{2 \pi r}, \quad\left(\text { for } r>r_{\mathrm{c}}\right)
$$

One of the differences between the suction vortex and Burgers vortex is whether it has a surface or not; the surface deformation of the fully developed suction vortex shows the following $1 / r^{2}$ dependence:

$$
\Delta h(r)=-\frac{1}{2 g}\left(\frac{\Gamma}{2 \pi r}\right)^{2}
$$

where $\Delta h(r)$ is the vertical deviation of the liquid position from the liquid at rest.

The second region is a core region that is defined by a large vorticity area near or below a funnel-shaped surface. The flow pattern and the vorticity distribution in the core region of the suction vortex remain unclear. However, the vortex theorem tells us that the vorticity is transported by the inward flow.

In other words, in the initial stage of vortex formation, the microscopic area that carries the vorticity is gathered towards the rotation axis.

Then, in the final stage of vortex formation, the macroscopic vortex is built up at the center of the rotation. From this point of view, the microscopic area that carries the vorticity can be treated as an elemental vortex, which is a virtual object.

Further, the core radius of the suction vortex is determined by the competition between the diffusion and transportation of the elemental vortices.

Recently, Andersen et al. [4,5] demonstrated the validity of Eqs. (3) and (4) in the potential flow region using a water-filled rotating container with a drain hole. They used the particle image velocimetry technique to show that the azimuthal velocity started to deviate from the $1 / r^{2}$ law near the core. Stepanyants et al. [6] numerically solved the Navier-Stokes equation and revealed not only the azimuthal velocity but also the radial velocity, vertical velocity, and surface deformation for three vortex cases characterized by the relative position between the vortex apex and the drain hole. 


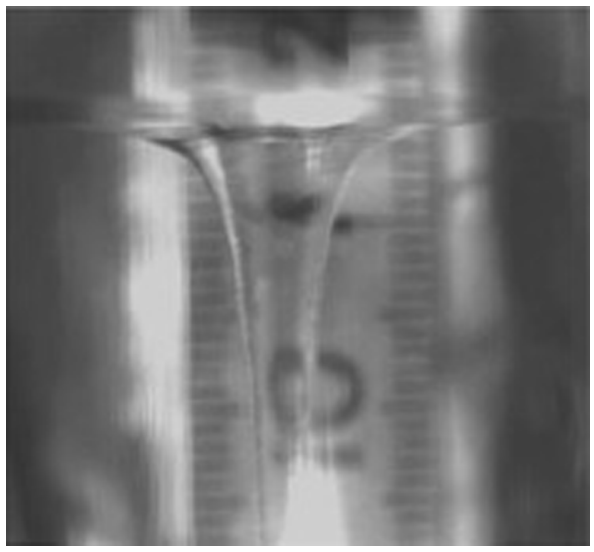

FIG. 1. Suction vortex in He II. The liquid temperature was $1.6 \mathrm{~K}$ and the turbine rotation speed was $5.8 \mathrm{rev} . / \mathrm{s}$. A $1-\mathrm{mm}$ scale ruler was set behind the vortex. The quantized vortex lines are too thin to be recognized in this photograph.

Here we explored the fundamental question, if the vorticity diffusion due to the kinematic viscosity plays an important role in forming a suction vortex, what will happen when we generate a suction vortex in inviscid fluids? Motivated by this question, we started to study a superfluid suction vortex [7].

In previous work, the turbine was driven by a cryogenic motor, and the centrifugal pump was carefully designed to generate the suction vortex both in normal fluid and superfluid phases. Figure 1 shows a typical photograph of a superfluid suction vortex with a surface shape that can be fitted to $1 / r^{2}$.

The macroscopic circulation dependence on motor speed for both normal fluid and superfluid were completely the same, revealing that our cryogenic agitating system could agitate superfluid and normal fluid just the same, that is, regardless of viscosity.

Our aim is to understand the core structure of the superfluid suction vortex.

\section{SUPERFLUID HE II AS A TOOL TO STUDY SUCTION VORTEX}

\section{A. Quantized vortex line in He II}

It is well known that the superfluid component of He II can be described by a macroscopic wave function $\Psi(\boldsymbol{r}, y)$ with quantum mechanical phase $\theta(\boldsymbol{r}, t)$, that is,

$$
\Psi(\boldsymbol{r}, t)=\Psi_{0} e^{i \theta(\boldsymbol{r}, t)},
$$

where $\boldsymbol{r}$ is the position in a condensate and $\left|\Psi_{0}\right|^{2}=\rho_{s} / \rho$ is the superfluid density fraction of He II. The velocity of the superfluid component $\boldsymbol{v}_{s}$ can be written by

$$
\boldsymbol{v}_{s}=\frac{\hbar}{m} \nabla \theta(\boldsymbol{r}, t),
$$

where $m$ is the mass of a ${ }^{4} \mathrm{He}$ atom and $\hbar=h / 2 \pi$ is Plank's constant. Equation (6) shows that the circulation $\oint_{\mathcal{C}} \boldsymbol{v}_{s} \cdot d \boldsymbol{l}$ of the superfluid around the contour $\mathcal{C}$ in a singly connected region must be zero. However, if the contour $\mathcal{C}$ encloses line defects of the condensate, then the circulation can take finite values. The smallest size of this defect is as small as the healing length of superfluid, which is about $0.1 \mathrm{~nm}$ for He II [8]. Because the macroscopic wave function must be single valued, $\theta(\boldsymbol{r})$ can only change by an integer multiple of $2 \pi$, which is called the quantization of the circulation. Moreover, because He II can only exist in low temperatures, the kinetic energy of the superfluid flow around the defect is limited to have the lowest value. Thus, the circulation of the superfluid 
component must be singly quantized as

$$
\kappa \equiv \frac{h}{m}=9.97 \times 10^{-8} \mathrm{~m}^{2} / \mathrm{s} .
$$

This constraint makes the vortex structure in a superfluid at the $T \rightarrow 0$ limit simpler; in contrast to the case of a vortex in a classical fluid with a continuous vorticity, in a superfluid, every vortex, including a macroscopically rotating flow and the turbulent flow, can be described by a complex of singly quantized vortices. However, when considering the liquid helium at finite temperature, a more intricate theory must be applied.

\section{B. Two-fluid model and second sound}

It is widely believed that hydrodynamics of He II can be described in terms of the two-fluid model proposed by Tisza [9] and Landau [10]. According to this model, superfluid behaves as a mixture of two fluids: viscous normal fluid and inviscid superfluid. In this model, we assign velocity fields $\boldsymbol{v}_{s}, \boldsymbol{v}_{n}$, and densities $\rho_{s}, \rho_{n}$, where the suffixes "s" and " $\mathrm{s}$ " indicate the normal fluid and superfluid components, respectively. Total density $\rho$ and the total flow density $\boldsymbol{j}$ can be written as

$$
\rho=\rho_{s}+\rho_{n}, \quad \boldsymbol{j}=\rho_{s} \boldsymbol{v}_{s}+\rho_{n} \boldsymbol{v}_{n},
$$

where the superfluid density fraction $\rho_{s} / \rho$ has a finite value only below the superfluid transition temperature $T_{\lambda}=2.17 \mathrm{~K}$ at saturated vapor pressure; this value smoothly increases as the fluid cools down and asymptotically approaches unity at temperatures below $1 \mathrm{~K}$. The equations of motion for two fluids in a vortex-free region are

$$
\begin{gathered}
\rho_{s}\left[\frac{\partial \boldsymbol{v}_{s}}{\partial t}+\left(\boldsymbol{v}_{s} \cdot \nabla\right) \boldsymbol{v}_{s}\right]=-\frac{\rho_{s}}{\rho} \nabla p+\rho_{s} \sigma \nabla T, \\
\rho_{n}\left[\frac{\partial \boldsymbol{v}_{n}}{\partial t}+\left(\boldsymbol{v}_{n} \cdot \nabla\right) \boldsymbol{v}_{n}\right]=-\frac{\rho_{n}}{\rho} \nabla p-\rho_{s} \sigma \nabla T+\eta \nabla^{2} \boldsymbol{v}_{n},
\end{gathered}
$$

where $T, p, \sigma$, and $\eta$ are temperature, pressure, entropy per unit mass, and viscosity of the normal fluid component, respectively. The first and second terms on the right-hand side of the equations are due to the spatial gradient of the chemical potential. One of the most significant results of the two-fluid model is predicting the existence of the second sound.

The second sound is a propagating wave of entropy or temperature in the bulk superfluid. The second sound velocity $c_{2}$ can be derived by linearizing Eqs. (9) and (10), neglecting the viscosity contribution, and assuming the equations of continuity for mass and entropy to hold as

$$
\frac{\partial \rho}{\partial t}=-\nabla \cdot \boldsymbol{j}, \quad \frac{\partial \sigma}{\partial t}=-\nabla \cdot\left(\rho \sigma \boldsymbol{v}_{n}\right)
$$

Straightforward calculation leads to the following relations:

$$
\begin{gathered}
\frac{\partial^{2} \rho}{\partial t^{2}}=\nabla^{2} p, \quad \frac{\partial^{2} \sigma}{\partial t^{2}}=\frac{\rho_{s}}{\rho_{n}} \sigma^{2} \nabla T, \\
\rho_{n} \frac{\partial}{\partial t}\left(\boldsymbol{v}_{n}-\boldsymbol{v}_{s}\right)=-\rho \sigma \nabla T,
\end{gathered}
$$

where the first equation in Eq. (12) corresponds to the ordinary compression wave in a viscous fluid and the second equation leads to a wave of temperature and entropy, which is specific to superfluid. Equation (13) shows that the counterflow velocity, $\boldsymbol{v}_{n s} \equiv \boldsymbol{v}_{n}-\boldsymbol{v}_{s}$, can be driven by the temperature gradient. Assuming a one-dimensional plane-wave solution for the pressure and temperature with 
linear dispersion relation $\omega=c k$, and using the definition of the adiabatic bulk modulus $K$ and the specific heat $C_{\mathrm{V}}$, one can find two values for $c$ :

$$
\begin{gathered}
c_{1}=\sqrt{\left(\frac{\partial p}{\partial \rho}\right)_{\sigma}}=\sqrt{\frac{K}{\rho},} \\
c_{2}=\sqrt{\frac{\rho_{s}}{\rho_{n}}\left(\frac{\partial T}{\partial \sigma}\right)_{\rho}}=\frac{\rho_{s}}{\rho_{n}} \frac{\sigma^{2} T}{C_{\mathrm{V}}},
\end{gathered}
$$

where $c_{1}$ is the speed of the pressure wave, in which both the normal and superfluid components oscillate in phase (called the first sound); and $c_{2}$ is the speed of the temperature wave, in which the normal and superfluid component oscillate out-of-phase, i.e., in which the local normal fluid density $\rho_{n} / \rho$ deviates from its thermal equilibrium value and propagates without changing its total mass density. Temperature dependencies of all thermodynamic quantities described above and the sound velocities at the saturated vapor pressure are summarized by Donnelly and Barenghi [11].

\section{Attenuation of first and second sound by quantized vortex}

In the previous section, we introduced the two-fluid model in a vortex-free region. Now, we take into account the vortex lines. According to a series of works done by Hall and Vinen [12-17], phonons and rotons are scattered by vortex lines. The energy and momentum of the rotons and phonons are transferred to the quantized vortex lines at each scattering event. Thus, from a macroscopic point of view, the motion of the quantized vortex line is affected by the normal fluid component, which consists of the rotons and phonons. Schwarz described the motion of a vortex filament $[18,19]$ by the following equation:

$$
\frac{d \boldsymbol{s}}{d t}=\boldsymbol{v}_{s}+\frac{\rho_{n}}{2 \rho} B \boldsymbol{s}^{\prime} \times\left(\boldsymbol{v}_{n}-\boldsymbol{v}_{s}\right)-\frac{\rho_{n}}{2 \rho} B^{\prime} \boldsymbol{s}^{\prime} \times\left[\boldsymbol{s}^{\prime} \times\left(\boldsymbol{v}_{n}-\boldsymbol{v}_{s}\right)\right],
$$

where $\boldsymbol{v}_{n}$ is the normal fluid velocity, $\boldsymbol{s}$ is the position of the vortex line, $\boldsymbol{s}^{\prime}$ is the tangential vector of the vortex line at $s$, and $B$ and $B^{\prime}$ are the mutual friction coefficients that describe the respective interaction between the vortex line and counterflow $\boldsymbol{v}_{n}-\boldsymbol{v}_{s}$. The first term in Eq. (16) can be understood as the vortex theorem for classical fluids, and the second and third terms are the results of the counterflow.

After these effects are taken into account, the equation of motion for two components in the presence of vortex lines becomes

$$
\begin{aligned}
& \rho_{s} \frac{\partial \boldsymbol{v}_{s}}{\partial t}=-\frac{\rho_{s}}{\rho} \nabla p+\rho_{s} \sigma \nabla T-\boldsymbol{F}_{\mathrm{ns}}, \\
& \rho_{n} \frac{\partial \boldsymbol{v}_{n}}{\partial t}=-\frac{\rho_{n}}{\rho} \nabla p-\rho_{s} \sigma \nabla T+\boldsymbol{F}_{\mathrm{ns}} .
\end{aligned}
$$

Here we neglect the higher-order term and the viscosity term in Eqs. (9) and (10) and $\boldsymbol{F}_{\mathrm{ns}}$ is called the mutual friction force per unit volume, described as

$$
\boldsymbol{F}_{\mathrm{ns}}=\kappa L \frac{\rho_{s} \rho_{n}}{2 \rho}\left\{B \boldsymbol{s}^{\prime} \times\left[\boldsymbol{s}^{\prime} \times\left(\boldsymbol{v}_{n}-\boldsymbol{v}_{s}\right)\right]+B^{\prime} \boldsymbol{s}^{\prime} \times\left(\boldsymbol{v}_{n}-\boldsymbol{v}_{s}\right)\right\},
$$

where $L$ is the average vortex line density in the vicinity of the sound propagating region. Because we are focusing on the propagation of sound, we only take into account the dissipative component of $\boldsymbol{F}_{\mathrm{ns}}$; the term $\boldsymbol{s}^{\prime} \times\left(\boldsymbol{v}_{n}-\boldsymbol{v}_{s}\right)$ does not contribute to the dissipation because the direction always becomes normal to $\boldsymbol{v}_{n}-\boldsymbol{v}_{s}$ and, in general, the coefficient $B^{\prime}$ is small compared with $B$ except near $T_{\lambda}$. As a result, the dissipation force in Eqs. (17) and (18) can be treated as

$$
\boldsymbol{F}_{\mathrm{ns}}=-B \kappa L \frac{\rho_{s} \rho_{n}}{2 \rho}\left(\boldsymbol{v}_{n}-\boldsymbol{v}_{s}\right) \sin ^{2} \theta
$$


where $\theta$ is the angle between vectors $\boldsymbol{s}^{\prime}$ and $\boldsymbol{v}_{n}-\boldsymbol{v}_{s}$. This sine-square law was experimentally confirmed by Snyder [20]. Straightforward calculation of the plane-wave solutions of Eqs. (17) and (18) show that the dispersion relation for the first sound is not affected by the existence of the vortex lines at all, but the dispersion relation for the second sound is affected as

$$
\omega^{2}-i \omega \frac{B \kappa L}{2} \sin ^{2} \theta=c_{2}^{2} k^{2},
$$

where $k$ is the complex wave number of the second sound. Finally, we have the second sound velocity $c_{2}^{\prime}$ and the attenuation of the second sound $\alpha_{v}$ due to the existence of the quantized vortex lines as

$$
\begin{gathered}
\alpha_{v}=-\operatorname{Im}(k)=\frac{B \kappa L}{4 c_{2}} \sin ^{2} \theta+\mathcal{O}\left(\frac{\kappa L}{c_{2}}\right)^{3}, \\
c_{2}^{\prime}=\operatorname{Re}\left(\frac{\omega}{k}\right)=c_{2}+\mathcal{O}\left(\frac{\kappa L}{c_{2}}\right)^{2} .
\end{gathered}
$$

Here the higher-order term in $c_{2}^{\prime}$ was neglected throughout our experiments.

\section{EXPERIMENTAL SETUP}

The setup of the experiment is shown in Fig. 2. To agitate He II in a cylinder, a six-blade turbine was rotated by a the cryogenic motor [7] which was mounted in the inner chamber. The driving system of the motor was a two-pole dc brushless motor of the out-runner type. The stator coils were wound with superconducting wire, and a sliding bearing was used to hold the turbine rotor shaft. The inner diameter of the inner chamber was $55 \mathrm{~mm}$, and the chamber had a side slit $(2.0 \mathrm{~mm})$ along the bottom plate and a hole $(5 \mathrm{~mm})$ at the center of the top plate. When the motor rotated, the inner chamber acted as a centrifugal pump, which caused the rotating flow in the chamber to eject the fluid from the slit and force it to return through the drain hole, producing a circulating loop. The fluid ejected from the side slit had a nonzero angular momentum as a result of the vorticity generated by the rotating flow in the cylinder. Details are shown in our previous paper [21]. The rotation speed of the turbine $\Omega / 2 \pi$ was measured directly by a Hall probe located under the rotor. We obtained a macroscopic circulation by means of two techniques. The first technique was to measure the surface deformation, which was done by taking photographs. The circulations, which corresponded to the circulating flow on the surface, were calculated by fitting their contours of the surface shape with Eq. (4). Note that this intuitive method was only valid when the angle of incidence at the interface of materials with different refractive indexes was negligibly small. In our experiment, the surface of the liquid helium (refractive index of 1.045) was observed through four layers of curved glass walls, namely, two walls for the liquid nitrogen Dewar vessel, and two walls for the liquid helium Dewar vessel. The refractive index and the thickness of the glass were 1.45 and $4 \mathrm{~mm}$, respectively. Thus, the larger the horizontal size of the vortex surface, the larger the apparent vortex size; in this case, the circulation of the vortex tended to be larger.

The second technique to measure the macroscopic circulation of the suction vortex was an ultrasonic circulation meter, which was an extended version of an ultrasonic flowmeter. Its principle for measuring the circulation is to measure the difference between the time of flight of the ultrasonic first sound pulses, propagating horizontally, with and against the direction of the circulating flow. Here, the azimuthal velocity profile was assumed to be $v_{\theta}(r)=\Gamma / 2 \pi r$, which was supported by the pressure balance between the centrifugal force and gravity at the surface with a profile described by Eq. (4). Moreover, it is important that the first sound is hardly affected by the quantized vortex line, as discussed in the previous section. Figure 3 shows the schematic drawing of our ultrasonic circulation meter setup. Here $2 R$ is the diameter of the experimental cylinder, point $\mathrm{O}$ indicates the center, and ultrasonic transducers are located at points $\mathrm{T}$ and $\mathrm{R}$. These transducers were made of lead zirconate titanate with a buffer layer to match the acoustic impedance so as to generate pulses with 
(a)
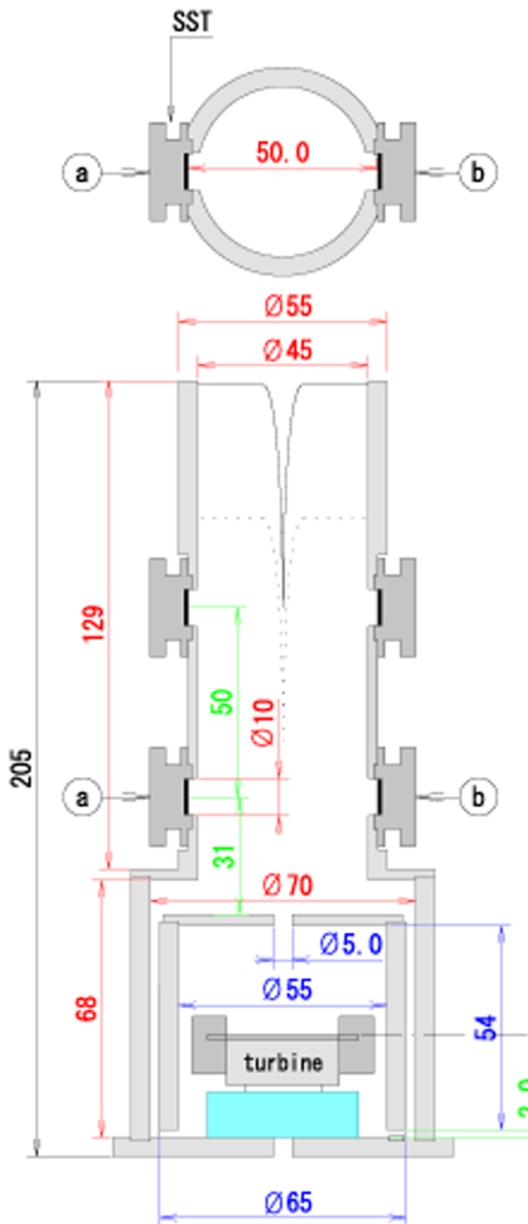

(b)

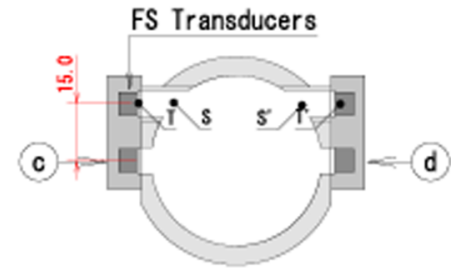

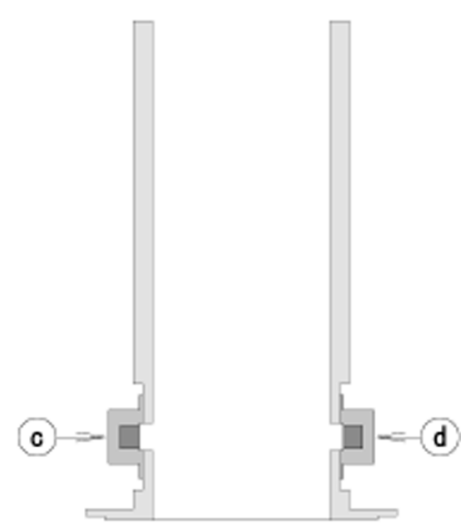

FIG. 2. Schematic of guide pipe, motor, and transducers. The pair of the transducers could be changed from ultrasonic transducers (FS transducers) to measure the first sound and metalized nucleopore membrane transducers for the second sound (SS transducers). The liquid surface was kept below the top edge of the guide pipe, and the edges of the surface were kept above the second sound cavity.

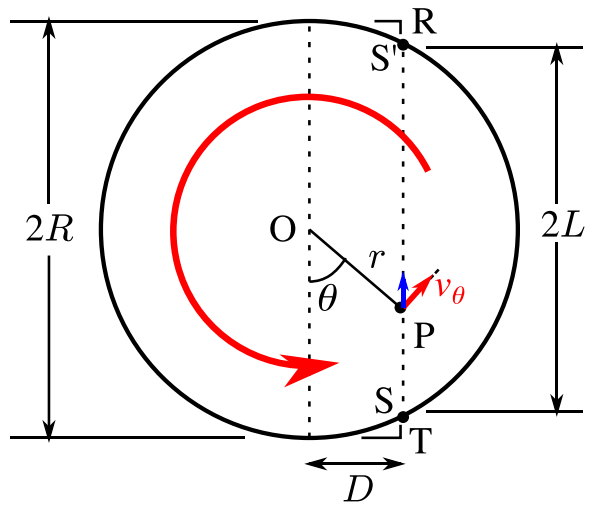

FIG. 3. Schematic of ultrasonic circulation meter. 
low power consumption. The operating frequency was $300 \mathrm{kHz}$, the pulse duration was 20 cycles, and the repetition time was $100 \mathrm{~ms}$. Before carrying out the flow measurements under rotation, we measured the first sound velocity at rest and confirmed that the first sound velocity perfectly agreed with well-established data [11]. The center of the active area of the transducers were offset by $D$ from the horizontal axis of symmetry. The estimated maximum azimuthal velocity on the propagating path was two orders smaller than the first sound velocity $c_{1}$ so that the propagating path could be treated as a straight line. When the wave front of the pulse was located at point $P$, the apparent sound velocity was affected by the azimuthal velocity $v_{\theta}(r)$. As shown in the Appendix, we numerically obtained the relation between the macroscopic circulation and the time of flight between $S$ and $S^{\prime}$ with and against the flow as

$$
\Gamma=A\left|\tau_{w}-\tau_{a}\right|,
$$

where $S$ and $S^{\prime}$ are the points where line T-R crosses the cylinder; $A=8.7615 \times 10^{4} \mathrm{~m}^{2} / \mathrm{s}^{2}$ for $D=15.0 \mathrm{~mm}$; and $\tau_{w}$ and $\tau_{a}$ are the time of flight with and against the flow, respectively.

The vorticity of the suction vortex was determined by measuring the attenuation of the second sound propagating across the vortex. The second sound was excited and detected using a metalized film pressure transducer with a membrane consisting of a gold-plated nucleopore filter with a nominal pore diameter of $0.4 \mu \mathrm{m}[22,23]$. A pair of transducers with surface areas $10 \mathrm{~mm}$ in diameter was located $31 \mathrm{~mm}$ above the orifice. The second sound standing wave resonance technique was used to determine the resonance frequency and quality factor of the second sound. The relation between the quality factor and the attenuation is described below. After we measured a standing wave resonance spectrum, we could obtain the resonance frequency $f_{m}$, the half width of half maximum $\Delta f_{m}$, and the peak height $A$, where $m$ is an integer that indicates the mode of the resonance. The quality factor is determined as $Q_{m}=f_{m} / \Delta f_{m}$. Suppose that the propagating second sound experiences the total attenuation $\tilde{\alpha}(\Omega)$. Then $\tilde{\alpha}$ consists of the rotation-independent component $\alpha_{0}$ that is mainly caused by the viscous damping and the attenuation caused by the quantum vortex lines, that is,

$$
\tilde{\alpha}(\Omega)=\alpha_{0}+\alpha_{v}(\Omega)
$$

In general wave theory, the attenuation can be described by $\pi / Q \lambda$, where $\lambda$ is the wavelength of the second sound. Therefore, in our case, the second sound attenuation under rotation $\Omega$ can be described by

$$
\tilde{\alpha}(\Omega)=\frac{\pi}{Q_{m}(\Omega) \lambda}
$$

and the attenuation at rest is given by

$$
\alpha_{0}=\frac{\pi \Delta f_{m}(0)}{c_{2}} .
$$

Finally, we have the relation between the quality factor of the second sound standing wave resonance and the attenuation due to the quantum vortex line as

$$
\alpha(\Omega)=\frac{\pi \Delta f_{m}(0)}{c_{2}}\left[\frac{Q_{m}(0)}{Q_{m}(\Omega)}-1\right] .
$$

If the vortex lines are assumed to be uniformly distributed and completely polarized in the cylinder, that is, $\sin ^{2} \theta$ in Eq. (22) is taken as unity, then the vortex line density $L(\Omega)$ can be revealed by the following equation:

$$
L(\Omega)=\frac{\pi t \Delta f_{m}(0)}{B \kappa}\left[\frac{Q_{m}(0)}{Q_{m}(\Omega)}-1\right] .
$$

Here the parameter $t$ takes a value of four if the vortex lines are uniformly distributed and completely polarized in the cylinder. If the vortex lines are fully turbulent, homogeneous, and isotropic, then $\sin ^{2} \theta$ in Eq. (22) becomes its spatial averaged value 2/3, and the parameter $t$ takes a value of six. 
(a)

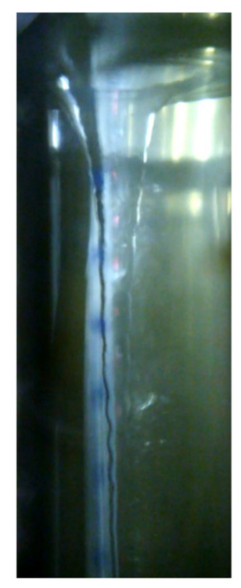

(b)

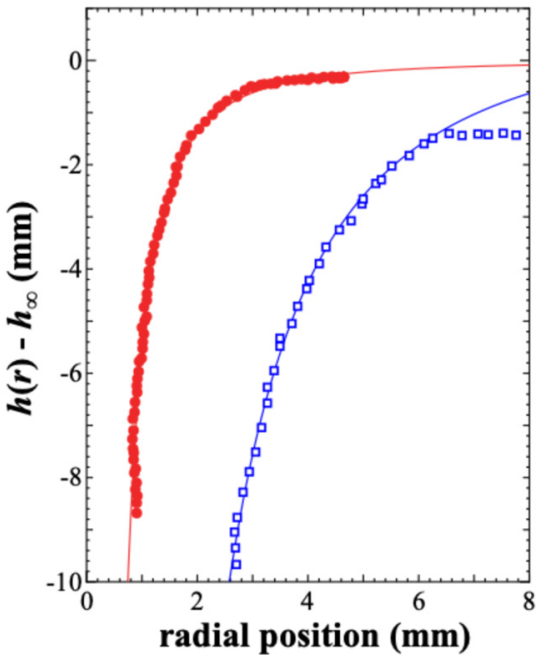

FIG. 4. (a) Photograph of typical surface deformation. (b) Surface profile; solid red circles and open blue squares represent 5.0 and $7.8 \mathrm{rev} . / \mathrm{s}$, respectively.

\section{RESULTS}

All experiments described below were carried out at $1.6 \mathrm{~K}$, the corresponding superfluid density fraction was $\rho_{s} / \rho=0.835$, and the liquid surfaces were below the top edge of the guide pipe.

\section{A. Determination of circulation by surface deformation}

The first experiment was carried out to obtain the macroscopic circulation $\Gamma$ by evaluating photographs of the deformed liquid surface $\Delta h(r)=h(r)-h_{\infty}$ under constant motor rotation speed. Figure 4(a) shows a typical image of the suction vortex with a clear funnel-shaped surface deformation. Although there were some small wavy structures, it was possible to fit their positions on the surface by Eq. (4), as shown in Fig. 4(b). The resulting circulation of the vortex at the surface was $\Gamma=(0.0020 \pm 0.0001) \mathrm{m}^{2} / \mathrm{s}$ at $5.0 \mathrm{rev} . / \mathrm{s}$. At $7.8 \mathrm{rev} . / \mathrm{s}$, the surface deformation started to deviate from Eq. (4) at $r=6 \mathrm{~mm}$. If we fit this position within a window of $r<6 \mathrm{~mm}$, then we obtain $\Gamma=(0.0094 \pm 0.000) \mathrm{m}^{2} / \mathrm{s}$. However, it should be noted that the horizontal width of the vortices at this speed was too large and the circulation might be overestimated due to optical effects.

\section{B. Determination of circulation by ultrasonic circulation meter}

Because the surface deformations due to the rotational flow were well described by Eq. (4), the ultrasonic circulation meter technique is valid for determining the circulation.

The circulation of the vortex is shown in Fig. 5(b). The results from the surface photography technique are denoted by empty squares. These data almost coincide with the experimental error, revealing that the circulation $\Gamma$ at the surface and in the bulk are almost the same; in other words, horizontal distribution of the circulation was small. The error is mainly due to the fact that the rotation of the turbine was not smooth at lower rotation speeds; in fact, it provided almost stop-andgo motion at $4 \mathrm{rev} . / \mathrm{s}$, so that the flow was not steady over the time span of each pulse.

\section{Second sound attenuation of suction vortex}

A third experiment was carried out to determine the vortex line density, which directly corresponds to the vorticity of the superfluid suction vortex. Figure 6(a) shows the power spectrum $P(f)$ of the standing wave resonance of the second sound at $1.6 \mathrm{~K}$ at rest. We obtained a 

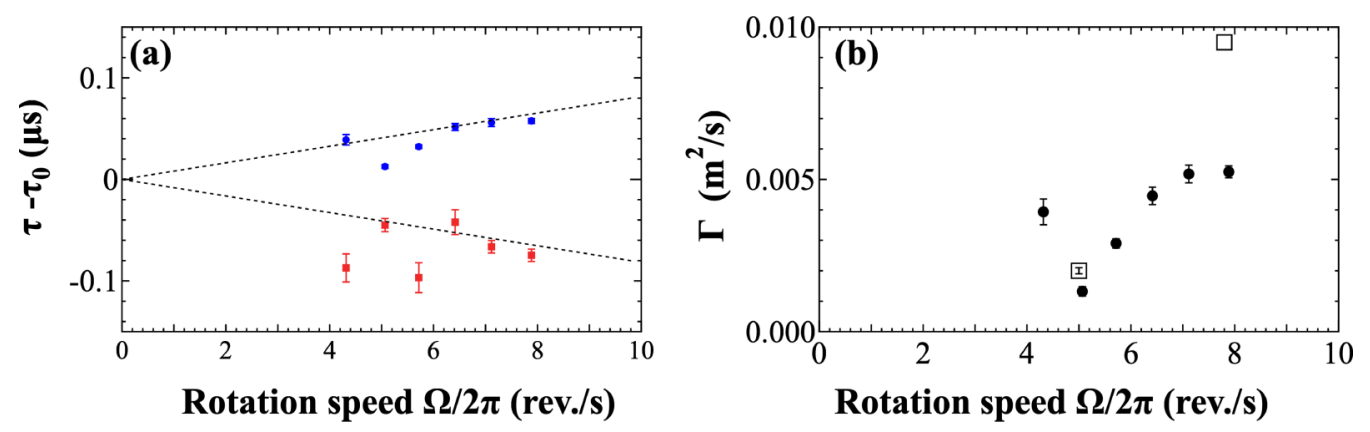

FIG. 5. Results of measurements with ultrasound circulation meter. (a) Time of flight of first sound pulse across suction vortex. Dotted lines are visual guides. (b) Calculated circulation. Open squares show the results from the surface deformation experiment.

sequence of resonance spectra, which contained more than 50 equally spaced resonance peaks, and each was successfully assigned a mode number $m$. The peak heights of each mode reflect the frequency dependence of our transducer. The mode number $m=67$ was selected to balance the trade-off between sensitivity and signal separation; the wavelength of corresponding second sound standing wave in the cavity was $1.5 \mathrm{~mm}$. Figure 6(b) shows the typical power spectra of the second sound standing wave resonance under rotation. The resonance frequencies shifted as the rotational speed was increased; a shift of $18 \mathrm{~Hz}$ was observed at $3.73 \mathrm{rev} . / \mathrm{s}$, and the corresponding change in the second sound velocity was $\Delta c_{2}=-0.023 \mathrm{~m} / \mathrm{s}$. This small change in the velocity could be interpreted as a temperature rise of approximately $8 \mathrm{mK}$, which is reasonable because the thermometer and the second sound resonator were separated by the thick wall of the guide pipe. The second sound resonance condition was held even under rotation. This means that the second sound could travel back and forth through the cavity many times, even when a rotational flow was present. All second sound resonance spectra in this report are reused from the previous report [21]. In that experiment, the surface shape of the vortex was slightly tilted and slightly moving. As a result, the fitting errors were too large. Here, we updated the fitting procedure to obtain more accurate data, as described below. We concentrate on the case in which the tip of the vortex surface did not penetrate into the second sound resonance cavity, which was labeled as case (i) in the previous report [24]. In
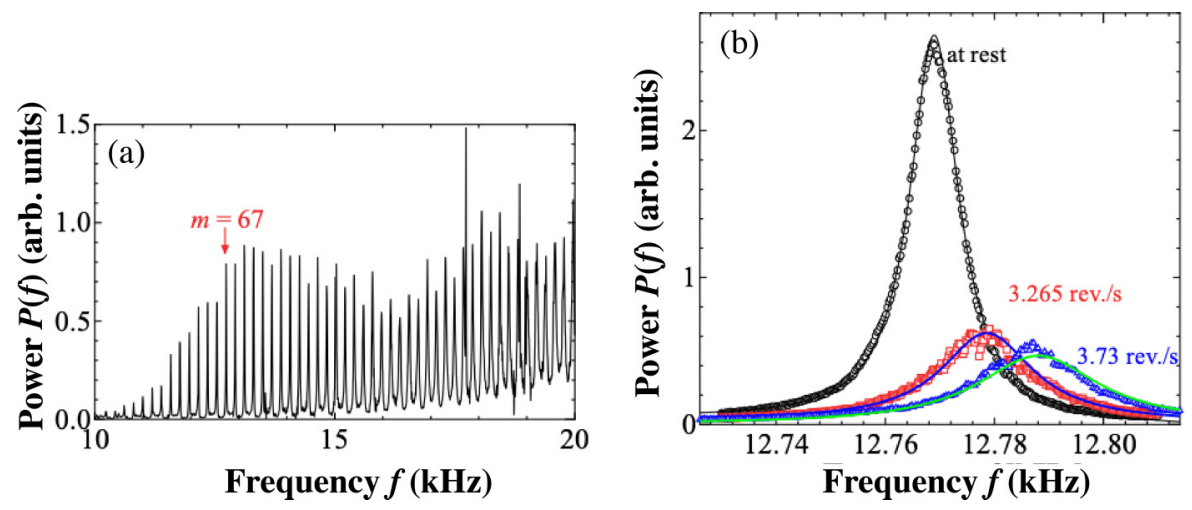

FIG. 6. (a) Power spectrum of second sound resonance at rest at $1.6 \mathrm{~K}$. (b) Typical power spectra of second sound resonance under rotation. Black circles, red squares, and blue triangles indicate the rotation speed at 0 , 3.265 , and 3.73 rev./s, respectively. Solid curves show the best fit. The small change in the resonance frequency could be interpreted as a temperature rise of approximately $8 \mathrm{mK}$. 


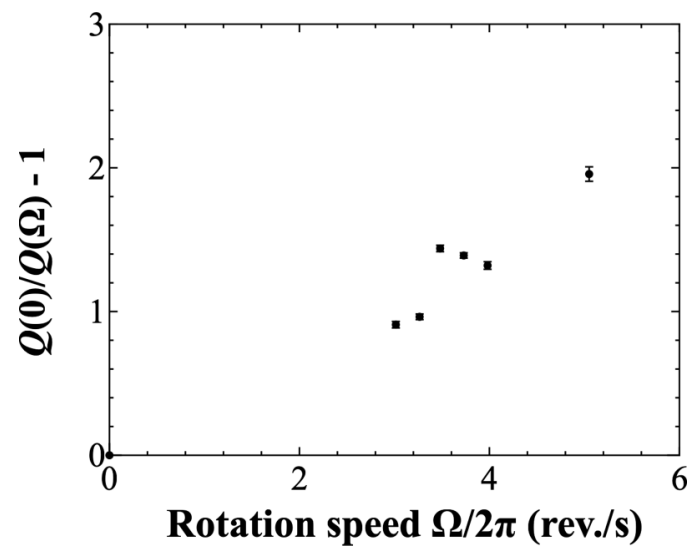

FIG. 7. Rotation speed dependence of damping factor $Q(0) / Q(\Omega)-1$.

this study, the following fitting model was applied:

$$
P(f)=\frac{A}{(\Delta f)^{2}+4\left(f-f_{r}\right)^{2}}+(\text { linear background }),
$$

where $A, \Delta f$, and $f_{r}$ are the amplitude, the full width at half maximum of the resonance, and the resonance frequency, respectively, and these parameters are treated as fitting parameters.

Now we show the attenuation of the second sound propagating through the core region of the suction vortex. Figure 7 shows the rotation speed of the motor dependence of the damping factor $Q(0) / Q(\Omega)-1$, demonstrating that the damping due to the quantized vortex lines monotonically increased with the rotation speed of the motor. However, it is not possible to apply Eq. (29) simply, because the vortex line distribution was not uniform. Thus, we need a model to extract the local vortex line density and the core radius of the superfluid suction vortex.

\section{DISCUSSION}

As we introduced in Sec. II C, the second sound is attenuated by both viscous attenuation $\alpha_{v}$ and the attenuation due to the quantized vortex lines in the resonance cavity. Now we consider the following model: All the quantized vortex lines were concentrated into the effective core region of the radius $R_{C}$, and the local vortex-line density in the effective core took a uniform value $L_{C}$.

Here the attenuation of the second sound due to the quantized vortex lines was $\alpha\left(L_{C}\right)$. Here the inter vortex-line spacing is much smaller than the wavelength of the second sound resonance. As such, the vortex core can be treated as a continuous dissipative region. We also assume that the radius of the second sound beam was thinner than the effective core radius, which is justified by the fact that the damping factors were very large at the finite rotation speed of motor; in other words, almost every partial wave emitted from the transducers and reflected at the transducers passed through the core. In this model, the second sound propagation can be treated in one dimension, which is shown in Fig. 8 schematically. Here we extend the second sound attenuation mechanism summarized in the Appendix of Ref. [25]. In our experimental setup, the length of the cavity was $2 R$. When the second sound is excited at resonant frequency, the waves reflected at the receiver will constructively interfere with the waves emitted from the transducer, giving the total measured amplitude under motor rotation as

$$
A\left(L_{C}\right)=a_{e} \sum_{n=1}^{\infty} e^{-\left[2 \alpha_{0} R+2 \alpha_{v}\left(L_{C}\right) R_{C}\right] n}, \quad A(0)=a_{e} \sum_{n=1}^{\infty} e^{-2 \alpha_{0} R} .
$$




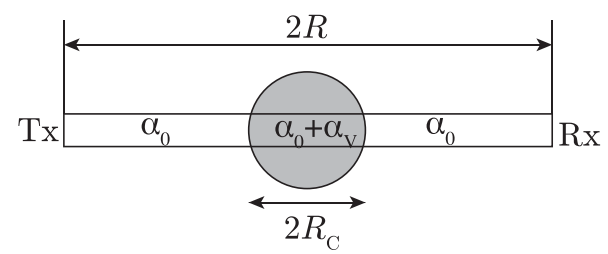

FIG. 8. One-dimensional model for second sound passing though the core. Tx and Rx correspond to the transmitter and the receiver, respectively, of the second sound. The size of the core was $2 R_{C}$.

Here $A(0)$ is the total amplitude at rest, and $a_{e}$ is the amplitude of the second sound excited at the transmitter. Because the damping over the distance $2 R$ is very small, Eqs. (31) can be reduced as

$$
A\left(L_{C}\right) \sim \frac{a_{e}}{2 \alpha_{0} R+2 \alpha_{v}\left(L_{C}\right) R_{C}}, \quad A(0) \sim \frac{a_{e}}{2 \alpha_{0} R} .
$$

Then the amplitude ratio becomes

$$
\frac{A\left(L_{C}\right)}{A(0)}=\frac{1}{1+\frac{\alpha_{v}\left(L_{C}\right)}{\alpha_{0}} \frac{R_{C}}{R}},
$$

which is equal to the ratio of the $Q$ value, $Q(\Omega) / Q(0)$. Because we assume that the fluid in the core region flows as a rigid body and the quantized vortex lines are polarized, the attenuation ratio can be written in the conventional form,

$$
\frac{\alpha_{0}}{\alpha_{v}}=\frac{4 \pi \Delta f}{B \kappa L_{C}} .
$$

Then, one finds that the local density of the vortex lines in the core is

$$
L_{C}=\frac{t \pi \Delta f}{B \kappa}\left[1-\frac{Q(0)}{Q(\Omega)}\right] \frac{R}{R_{C}} .
$$

Here the parameter $t$ represents the configuration of the vortex lines and takes a value between four and six. To estimate the effective core radius $R_{C}$, we assume that the quantized vortex lines in the core are straight and orientated vertically, or, in other words, that the flow in the core is undergoing rigid-body rotation. In this case, the vortex-line density can be treated as

$$
L_{C}=\Gamma / \kappa \pi R_{C}^{2},
$$

where $\Gamma$ is measured by the ultrasound circulation meter. By equating Eqs. (35) and (36), we obtain

$$
R_{C}=\left\{\frac{4 \pi^{2} R \Delta f}{B \Gamma}\left[1-\frac{Q(0)}{Q(\Omega)}\right]\right\}^{-1} .
$$

The effective core radius $R_{C}$ was calculated to be almost one-tenth (about $0.1 \mathrm{~mm}$ for $6 \mathrm{rev} . / \mathrm{s}$ ) of the wavelength of the second sound in the cavity, which is too narrow for the second sound resonator to be able to detect quantized vortex lines. Thus, the model assuming the straight vortex lines was not appropriate for describing experimental results. The main reason for this discrepancy is that the local vortex line density $L_{C}$ was overestimated, and the reasons for this are described below.

Since we have not been able to determine the effective radius of the superfluid suction vortex thus far, we adopt the radius of the drain hole $R_{d}$ as the effective radius instead, as it is of the typical length scale for this suction vortex. In this case, the local vortex-line density can be obtained from the experimental value of $\Gamma_{z}$ which was measured by the ultrasound circulation meter as

$$
L_{C}^{\mathrm{fs}}=\frac{\Gamma_{z}}{\kappa \pi R_{d}^{2}} .
$$



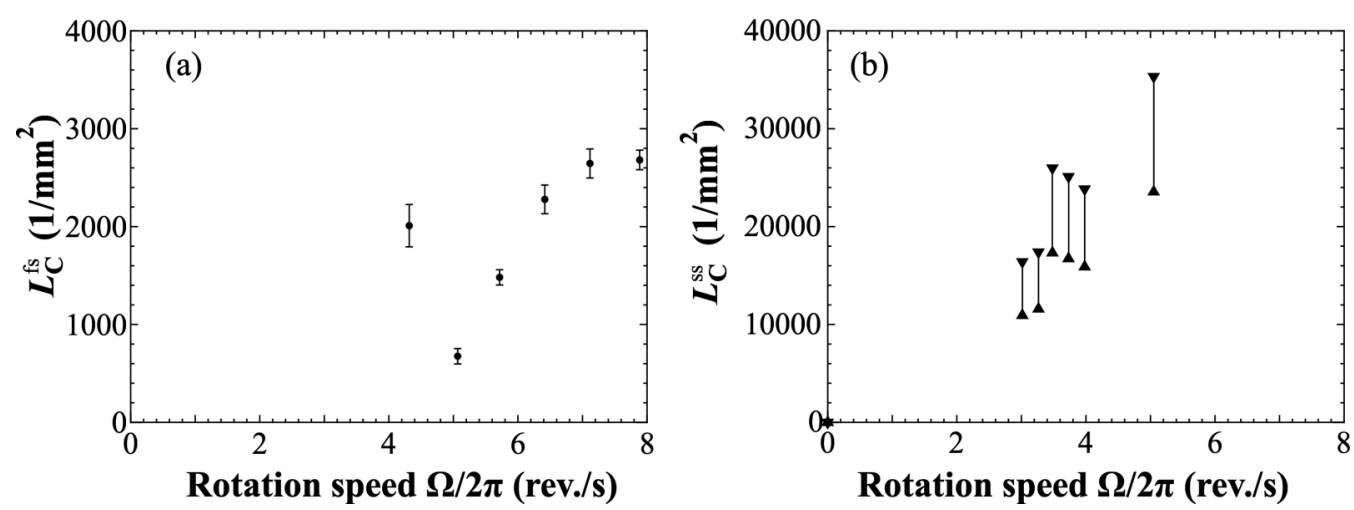

FIG. 9. Estimated local vortex-line densities: (a) From ultrasound circulation meter and (b) from second sound resonance. The upward and downward facing triangles represent the lower and upper limits, respectively.

Here the macroscopic circulation $\Gamma_{z}$ is now treated as a line integral of the net circulating flow around the core and the integral must be carried out in the horizontal plane including the ultrasound transducers, that is,

$$
\Gamma_{z}=\oint_{r>R_{d}} v_{\theta}(r) d r
$$

In other words, $\Gamma_{z}$ corresponds to the sum of the vertical components of the vorticity of the quantized vortex lines. Additionally, the local vortex-line density in the core region can be obtained from the second sound resonance by,

$$
L_{C}^{\mathrm{ss}}=\frac{t \pi \Delta f}{B \kappa}\left[1-\frac{Q(0)}{Q(\Omega)}\right] \frac{R}{R_{d}} .
$$

Here $L_{C}^{\text {ss }}$ is not the density of vertical vortex lines in the circular region of radius $R_{C}$ (Fig. 8), but the vortex-line density in a cylinder of radius $d$ and height $h$. Additionally, $h$ is the effective diameter of the transducer, and $R_{d}<h$. A significant difference between these two quantities appears when the vorticity vector involves nonvertical components.

The calculated results are shown in Figs. 9(a) and 9(b). Here, in Fig. 9(a), the upward facing triangles represent the lower limit, i.e., $t=4$, and the downward facing triangles represent the upper limit, i.e., $t=6$. Although the data points are scattered, we can see that the $L_{C}^{\mathrm{ss}}$ and $L_{C}^{\text {us }}$ tend to increase with increasing motor rotation speed. We can also see that the vortex-line density $L_{C}^{\mathrm{fs}}$ calculated from the macroscopic circulation measured by the ultrasound circulation meter is only about one tenth the vortex-line density $L_{C}^{\text {ss }}$ measured by the second sound attenuation measurement. To explain this discrepancy, we propose that the majority of the vortex lines in the core of the suction vortex do not contribute to the macroscopic rotation about the vertical axis, i.e., the vortex lines are inclined in the horizontal direction. This would be consistent with the general property that vortex lines are preferentially perpendicular to the external flow. This picture is also in agreement with the numerical simulation by Inui et al. [26], who solved the motion of the quantized vortex lines under the influence of the vertical normal fluid flow.

Although the conditions in that study were rather artificial, it is reasonable to think that the normal fluid suction flow enhanced the horizontal component of the vorticity vector in a narrow area around the axis of rotation.

In addition, in order to clarify the nature of the suction vortex, it is necessary to precisely determine the core size. Therefore, we plan to measure the attenuation of a second sound that does not pass through the center of rotation and to perform a vortex line visualization using fine particles. 


\section{SUMMARY AND FUTURE PROSPECTS}

We have studied the suction vortex in He II directly driven by a cryogenic motor. The macroscopic circulation of the suction vortex was measured by surface visualization and the pulsed first sound time-of-flight measurement. Then, the density of the quantized vortex lines were measured using the second sound resonance technique. By combining these three techniques, we propose that the majority of the vortex lines in the core of the suction vortex do not contribute to the macroscopic rotation about the vertical axis. This implies that these vortex lines are inclined in the horizontal direction. This would be consistent with the general property that vortex lines are preferentially perpendicular to the external flow. We are now improving our agitating system to clarify the properties of the suction vortex in the low-speed rotation region, below $3 \mathrm{rev} . / \mathrm{s}$, and are also improving the accuracy of the ultrasound circulation measurement.

Finally, we comment on the core structure that was observed by Andersen et al. [4,5]. They found that the characteristic stream lines spring up from the drain hole, which followed the Ekman layer at the outer region but spiraled upward around the axis of symmetry until the flow turned and then spiraled down toward the drain hole. The existence of these spiral stream lines may attract academic interest in the context of the Ekman pumping mechanism in oceanic dynamics and the cell structure around the core of tornado. This structure cannot be observed by our ultrasonic circulation meter and the second attenuation technique. Thus, our next step is to apply a vortex line imaging technique, such as particle tracking velocimetry or particle image velocimetry, to clarify the characteristic stream.

\section{ACKNOWLEDGMENTS}

The authors thank S. Inui and M. Tsubota for the technical assistance for the calculations discussed in the Appendix. The research was supported by the Japan Society for the Promotion of Science; KAKENHI Grants No. 15H03694, No. 17K18761, and No. 20K03865; and the Osaka City University, Strategic Research Grant No. 2019/2020 for top-priority research.

\section{APPENDIX: PROPAGATION OF SOUND WAVE THROUGH ROTATING FLOW}

Here we consider ordinary sound propagating through classical and compressive fluids with a steady but inhomogeneous flow field $\boldsymbol{u}(\boldsymbol{r})$ in two dimensions. According to Landau and Lifshitz [27], the position of the propagating wave $\boldsymbol{r}=(x, y)$ is described by

$$
\dot{r}=c \frac{k}{|k|}+u(r)
$$

where $\boldsymbol{k}=\left(k_{x}, k_{y}\right)$ is the wave vector of the sound. The important point in this system is that not only the wave velocity $\dot{r}$ but the wave vector is also affected by the flow field as

$$
\dot{\boldsymbol{k}}=-(\boldsymbol{k} \cdot \nabla) \boldsymbol{u}(\boldsymbol{r})-\boldsymbol{k} \times[\nabla \times \boldsymbol{u}(\boldsymbol{r})],
$$

where $c$ is the velocity of sound in the fluid at rest. Here we take a Rankine vortex with a circulation of $\Gamma$ and core radius of $r_{C}$ as a simplified model for our suction vortex. The flow velocity of the Rankine vortex is

$$
\begin{gathered}
|\boldsymbol{u}(\boldsymbol{r})|= \begin{cases}\frac{\Gamma}{2 \pi r} & \left(\text { for } r \geqslant r_{C}\right), \\
\frac{\Gamma}{2 \pi r_{C}} \frac{r}{r_{C}} & \left(\text { for } r<r_{C}\right),\end{cases} \\
\boldsymbol{u}(\boldsymbol{r})=|\boldsymbol{u}(\boldsymbol{r})|\left(\begin{array}{c}
-y / r \\
x / r
\end{array}\right),
\end{gathered}
$$




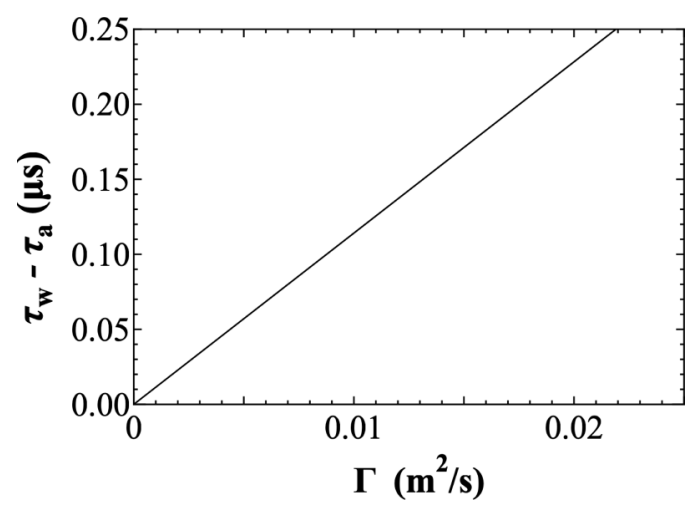

FIG. 10. Time-of-flight calculation for Rankine vortex of circulation $\Gamma$. The first sound velocity $c$ was set to $234.53 \mathrm{~m} / \mathrm{s}$ at $1.6 \mathrm{~K}$, and the operating frequency was set to $f=300 \mathrm{kHz}$. This calculation is only valid if the core size is smaller than $15 \mathrm{~mm}$.

where $r=\sqrt{x^{2}+y^{2}}$. The time evolution of the wave vector becomes

$$
\dot{k}=\frac{\Gamma}{2 \pi r^{4}}\left(\begin{array}{cc}
-2 x y & x^{2}-y^{2} \\
x^{2}-y^{2} & 2 x y
\end{array}\right)\left(\begin{array}{l}
k_{x} \\
k_{y}
\end{array}\right)
$$

for the free vortex region $\left(r>r_{C}\right)$, and

$$
\dot{k}=\frac{\Gamma}{2 \pi r_{C}^{2}}\left(\begin{array}{c}
-k_{y} \\
k_{x}
\end{array}\right)
$$

for the core region $\left(r<r_{C}\right)$. One can directly solve Eqs. (A1) and (A2) numerically by setting $\Gamma, r_{C}$, and the initial and boundary conditions. Now we describe the ultrasonic circulation meter described in Sec. IV A. In this case, we used $c=234.53 \mathrm{~m} / \mathrm{s}$ and $f=300 \mathrm{kHz}$, and the wave packets were emitted from the point $(-15 \mathrm{~mm},-22.5 \mathrm{~mm})$. As a result, we found that the trajectories were hardly affected by the rotating flow; however, the time-of-flight difference between the wave packet traveling with flow $\tau_{w}$ and against the flow $\tau_{a}$ linearly depended on the circulation: $\Gamma=A\left|\tau_{w}-\tau_{a}\right|$, which is shown in Fig. 10. From this principle, we could estimate the macroscopic circulation by means of measuring the time of flight of the first sound pulses. At the given conditions, $A=8.7615 \times 10^{4} \mathrm{~m}^{2} / \mathrm{s}^{2}$.

[1] J. M. Burgers, A mathematical model illustrating the theory of turbulence, Adv. Appl. Mech. 1, 171 (1948).

[2] A. H. Shapiro, Bath-tub vortex, Nature (London) 196, 1080 (1962).

[3] M. Sibulkin, A note on the bathtub vortex, J. Fluid Mech. 14, 21 (1962).

[4] A. Andersen, T. Bohr, B. Stenum, J. J. Rasmussen, and B. Lautrup, Anatomy of a Bathtub Vortex, Phys. Rev. Lett. 91, 104502 (2003).

[5] A. Andersen, T. Bohr, B. Stenum, J. J. Rasmussen, and B. Lautrup, The bathtub vortex in a rotating container, J. Fluid Mech. 556, 121 (2006).

[6] Y. A. Stepanyants and G. H. Yeoh, Stationary bathtub vortices and a critical regime of liquid discharge, J. Fluid Mech. 604, 77 (2008).

[7] H. Yano, K. Ohyama, K. Obara, and O. Ishikawa, Observation of the spiral flow and vortex induced by a suction pump in superfluid ${ }^{4} \mathrm{He}$, J. Phys.: Conf. Ser. 969, 012002 (2018).

[8] G. G. Ihas and F. Pobell, Correlation length, finite-geometry effects, and universality in pressurized superfluid helium near $t_{\lambda}$, Phys. Rev. A 9, 1278 (1974). 
[9] L. Tisza, Transport phenomena in Helium-II, Nature (London) 141, 913 (1938).

[10] L. Landau, Theory of the superfluidity of Helium-II, Phys. Rev. 60, 356 (1941).

[11] R. Donnelly and C. Barenghi, The observed properties of liquid helium at the saturated vapor pressure, J. Phys. Chem. Ref. Data 27, 1217 (1998).

[12] W. F. Vinen, Mutual friction in a heat current in liquid helium II, I. Experiments on steady heat currents, Proc. R. Soc. A 240, 114 (1957).

[13] W. F. Vinen, Mutual friction in a heat current in liquid helium II, II. Experiments on transient effects, Proc. R. Soc. A 240, 128 (1957).

[14] H. E. Hall and W. F. Vinen, The rotation of liquid heium II: Theory of mutual friction in uniformly rotating helium II, Proc. R. Soc. A 238, 215 (1956).

[15] H. E. Hall and W. F. Vinen, The rotation of liquid helium II, I. experiments on the propagation of second sound in uniformly rotating Helium-II, Proc. R. Soc. A 238, 204 (1956).

[16] W. F. Vinen, Mutual friction in a heat current in liquid helium II, III. Theory of the mutual friction, Proc. R. Soc. A 242, 493 (1957).

[17] W. F. Vinen, Mutual friction in a heat current in liquid helium II, IV. Critical heat currents in wide channels, Proc. R. Soc. A 243, 400 (1958).

[18] K. W. Schwarz, Three-dimensional vortex dynamics in superfluid ${ }^{4} \mathrm{He}$ : Line-line and line-boundary interactions, Phys. Rev. B 31, 5782 (1985).

[19] K. W. Schwarz, Three-dimensional vortex dynamics in superfluid ${ }^{4} \mathrm{He}$ : Homogeneous superfluid turbulence, Phys. Rev. B 38, 2398 (1988).

[20] H. A. Snyder and Z. Putney, Angular dependence of mutual friction in rotating helium-II, Phys. Rev. 150, 110 (1966).

[21] I. Matsumura, K. Ohyama, K. Sato, K. Obara, H. Yano, and O. Ishikawa, Observation of second sound attenuation across a superfluid suction vortex, J. Low Temp. Phys. 196, 204 (2019).

[22] W. M. Saslow, Superleak second-sound transducers, Phys. Rev. B 27, 588 (1983).

[23] M. Liu, Mechanical detectors of second sound, Phys. Rev. B 29, 2833 (1984).

[24] In case (ii) in the previous report [21], the tip of the vortex tube penetrated into the cavity. Clearly, the observed damping factors for case (ii) were much higher than for case (i) at a higher rotation speed, which means that a large second sound damping occurred due to scattering at the liquid-vacuum interface, and the damping mechanism was not necessarily due to the momentum transfer between the quantized vortex and the normal fluid component. This case may be important for understanding the second sound propagation, but this topic is outside the scope of this paper.

[25] S. Babuin, M. Stammeier, E. Varga, M. Rotter, and L. Skrbek, Quantum turbulence of bellows-driven ${ }^{4} \mathrm{He}$ superflow: Decay, Phys. Rev. B 86, 134515 (2012).

[26] S. Inui, T. Nakagawa, and M. Tsubota, Bathtub vortex in superfluid ${ }^{4} \mathrm{He}$, Phys. Rev. B 102, 224511 (2020).

[27] L. D. Landau and E. M. Lifshitz, Fluid Mechanics, 2nd ed. (Pergamon Press, London, 1987). 